\title{
A CRITERION FOR HYPERBOLICITY
}

\author{
by MICHAEL BATTY
}

(Received 12th May 1997)

\begin{abstract}
The usual definition of hyperbolicity of a group $G$ demands that all geodesic triangles in the Cayley graph of $G$ should be thin. Using the theorem that a subquadratic isoperimetric inequality implies a linear one, we show that it is in fact only necessary for all triangles from a given combing to be thin, thus giving a new criterion for hyperbolicity of finitely presented groups.
\end{abstract}

1991 Mathematics subject classification: $20 \mathrm{~F} 32$.

\section{Slim triangles}

Given a group $G$, the Cayley graph $\Gamma_{S}(G)$ of $G$ with respect to a generating set $S$ of $G$ is the graph whose vertex set is $G$ and whose edge set is $\{(g, g s) \mid g \in G, s \in S\}$. Given a path $p$ in $\Gamma_{s}(G)$, we write $l(p)$ for the number of edges in $p$. If $p$ originates at the identity of $G$ then we write $\bar{p}$ for the group element at the terminus of $p$ (i.e. $\bar{p}$ is the group element represented by the word $p$ in $S$ ).

Definition 1.1. A triangle in a group $G$ is the data

$$
\left(g_{1}, g_{2}, g_{3}, \theta_{12}, \theta_{23}, \theta_{31}\right) \text {, }
$$

where $g_{1}, g_{2}$, and $g_{3}$ are elements of $G$ called the vertices of the triangle and $\theta_{i j}$ is a path in the Cayley graph of $G$ from $g_{i}$ to $g_{j}$ (called a side of the triangle). If the sides are geodesic paths, the triangle is said to be geodesic.

For a triangle $\Delta$ as above, we denote by $\partial \Delta$ the loop $\theta_{12} * \theta_{23} * \theta_{31}$, called the boundary of $\Delta$ and we write $\pi(\Delta)$ for $l(\partial \Delta)$, the perimeter of $\Delta$.

The following definition is based on the familiar geodesic case.

Definition 1.2. Let $\delta \geq 0$. A triangle $\Delta$ in $G$ is $\delta$-slim if for each of the three sides $\theta$ of $\Delta$ and for all $t \in \theta, d\left(t, \theta^{\prime} \cup \theta^{\prime \prime}\right) \leq \delta$, where $\theta^{\prime}$ and $\theta^{\prime \prime}$ are the other two sides of $\Delta$.

If there exists a finite generating set $S$ of a group $G$ and $\delta \geq 0$ such that all geodesic triangles in $\Gamma_{s}(G)$ are $\delta$-slim then we say that $G$ is hyperbolic. In fact hyperbolicity of groups does not depend on the finite generating set chosen. Also note that it is a 


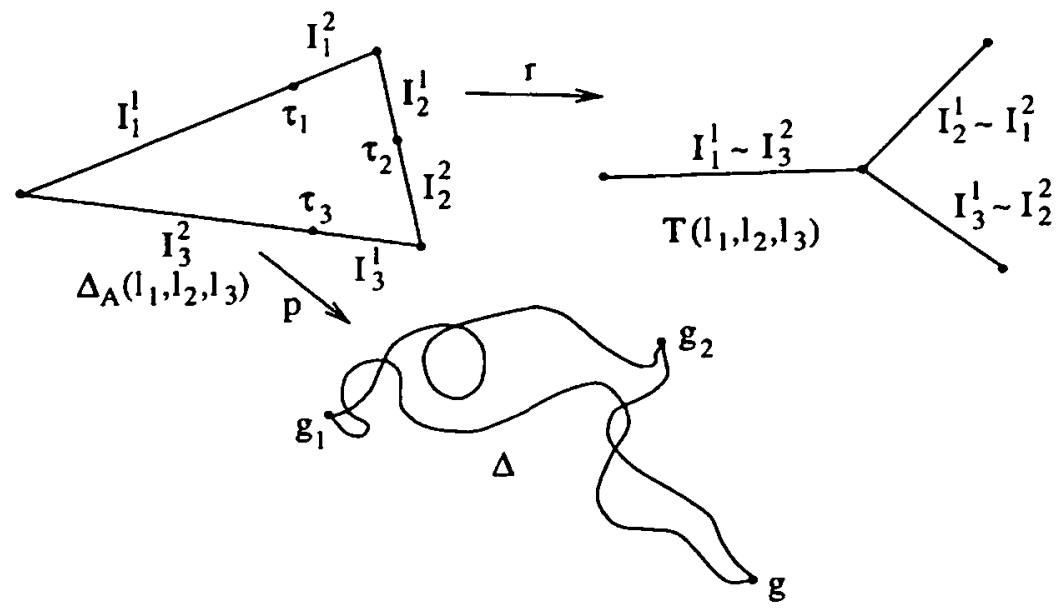

FIGURE 1: Collapsing a Triangle

consequence of the definitizon that all hyperbolic groups are finitely presented (these facts are proved in [10] and [4]).

\section{Thin triangles}

Let $l_{1} \geq 0, l_{2} \geq 0$ and $l_{3} \geq 0$ be real numbers satisfying the triangle inequality (in all possible permutations) and let $I_{1}, I_{2}$ and $I_{3}$ be the real intervals $\left[0, l_{1}\right],\left[0, l_{2}\right]$ and $\left[0, l_{3}\right]$ respectively with endpoints $i_{1}, t_{1}, i_{2}, t_{2}, i_{3}$ and $t_{3}$. Let $I\left(l_{1}, l_{2}, l_{3}\right)$ be the disjoint union of $I_{1}, I_{2}$ and $I_{3}$. Then we define the abstract triangle $\Delta_{A}\left(l_{1}, l_{2}, l_{3}\right)$ to be the quotient space of $I\left(l_{1}, l_{2}, l_{3}\right)$ by identifying $t_{1}$ with $i_{2}, t_{2}$ with $i_{3}$ and $t_{3}$ with $i_{1}$. Now there exist points $\tau_{1} \in I_{1}, \tau_{2} \in I_{2}$ and $\tau_{3} \in I_{3}$ with the following property: Suppose that $\tau_{1}$ divides $I_{1}$ into segments $I_{1}^{1}$ and $I_{1}^{2}, I_{2}$ into $I_{2}^{1}$ and $I_{2}^{2}$ and $I_{3}$ into $I_{3}^{1}$ and $I_{3}^{2}$ as in Figure 1. Then the length $l\left(I_{1}^{1}\right)=l\left(I_{3}^{2}\right), l\left(I_{2}^{1}\right)=l\left(I_{1}^{2}\right)$ and $l\left(I_{3}^{1}\right)=l\left(I_{2}^{2}\right)$. So we may isometrically identify $I_{1}^{1}$ with $I_{3}^{2}, I_{2}^{1}$ with $I_{1}^{2}$ and $I_{3}^{1}$ with $I_{2}^{2}$ as in Figure 1 to obtain a quotient space of $\Delta_{1}\left(l_{1}, l_{2}, l_{3}\right)$ called the tripod $T\left(l_{1}, l_{2}, l_{3}\right)$. We denote the quotient map by $r$. Then $r\left(I_{1}\right) \cap r\left(I_{2}\right) \cap r\left(I_{3}\right)$ is a single point, which we call the fork of the tripod.

Definition 2.1. We say that a triangle $\left(g_{1}, g_{2}, g_{3}, \theta_{12}, \theta_{23}, \theta_{31}\right)$ in a group is proper if the lengths of its sides satisfy the triangle inequality, i.e. for all $i \neq j \neq k$ with $1 \leq i, j, k \leq 3$,

$$
l\left(\theta_{i j}\right) \leq l\left(\theta_{j k}\right)+l\left(\theta_{k i}\right)
$$

For example, geodesic triangles are proper. Let $\Delta=\left(g_{1}, g_{2}, g_{3}, \theta_{12}, \theta_{23}, \theta_{31}\right)$ be a proper triangle in a group $G$, with $l\left(\theta_{12}\right)=l_{1}, l\left(\theta_{23}\right)=l_{2}$ and $l\left(\theta_{31}\right)=l_{3}$. If $x$ is a real 
number, denote by $[x]$ the greatest integer not exceeding $x$. There exists a map $p: I\left(l_{1}, l_{2}, l_{3}\right) \rightarrow G$ such that for all $x \in I_{1}$ with $0 \leq x \leq l_{1}, p(x)=\theta_{12}([x])$, for all $x \in I_{2}$ with $0 \leq x \leq l_{2}, p(x)=\theta_{23}([x])$, for all $x \in I_{3}$ with $0 \leq x \leq l_{3}, p(x)=\theta_{31}([x])$ and $p$ induces a map $p: \Delta_{A}\left(l_{1}, l_{2}, l_{3}\right) \rightarrow G$ on passage to the quotient.

Definition 2.2. Let $\delta \geq 0$. We say that a proper triangle $\Delta$ is $\delta$-thin if for all $t \in T\left(l_{1}, l_{2}, l_{3}\right), \operatorname{diam}\left(p\left(r^{-1}(t)\right)\right) \leq \delta$.

We next give a lemma describing the area of thin triangles, where area is in the following sense. Let $G=\langle S \mid R\rangle$ be a finitely presented group. Then recall that a word in $S$ is equal to the identity in $G$ if and only if there exist words $u_{i}$ in $S$ for $1 \leq i \leq n$ such that, in the free group $F(S)$ generated by $S$,

$$
w=\prod_{i=1}^{n} u_{i} r_{i} u_{i}^{-1}
$$

where for all $i$ with $1 \leq i \leq n$ either $r_{i} \in R$ or $r_{i}^{-1} \in R$.

Definition 2.3. With $G$ as above, let $w$ be a word in $S$ which is equal to the identity in $G$. Then the area of $w, A(w)$, is defined to be

$$
\min \left\{n \in \mathbb{N} \mid w=\prod_{i=1}^{n} u_{i} r_{i} u_{i}^{-1} \text { in } F(S)\right\} .
$$

We now describe an equivalent formulation of area which is more geometric and suited to our methods.

Definition 2.4. A paired alphabet is a finite set $S$ together with an involution $f: S \rightarrow S$. We usually write $f(s)=s^{-1}$.

For example, an inverse closed set of generators of a group is a paired alphabet, where the involution is the group inverse.

Definition 2.5. A map is a finite, planar, oriented, connected and simply connected combinational 2-complex. We say that a map $M$ is a diagram over a paired alphabet $S$ if every edge $e$ of $M$ has a label $\phi(e) \in S$ such that $\phi\left(e^{-1}\right)=(\phi(e))^{-1}$.

The definition of a map ensures that it has a well defined boundary path. Note that every path in a diagram over $S$ is labelled by a word in $S$.

Definition 2.6. A van Kampen diagram over a group $G=\langle S, R\rangle$ is a diagram $M$ over $S$ such that for all faces $f$ of $M$ the label of the boundary path of $f$ is labelled by some $r^{ \pm 1}$ with $r \in R$. The area of such a diagram is the number of its faces. 
Proposition 2.7 (van Kampen's lemma). Let $G=\langle S, R\rangle$ be a finitely presented group and let $w$ be a word in $S$. Then $\bar{w}=1_{G}$ if and only if there exists a van Kampen diagram. over $G$ with boundary labelled by $w$.

See [10] for a proof of van Kampen's lemma. We define the Dehn function of $G$ to be the function $D: \mathbb{N} \rightarrow \mathbb{N}$ given by $D(n)=\max \{A(w)\}$ where the maximum is taken over all words of length at most $n$ in $S$ such that $\bar{w}=1_{G}$.

Proposition 2.8. Let $G$ be a group and let $\delta \geq 0$. Then there exists a linear function $y: \mathbb{N} \rightarrow \mathbb{N}$ such that if $\Delta$ is a $\delta$-thin triangle in $G$ then $A(\Delta) \leq y(\pi(\Delta))$.

Proof. Suppose that $\Delta$ has side lengths $l_{1}, l_{2}$ and $l_{3}$. Let $f$ be the fork of $T\left(l_{1}, l_{2}, l_{3}\right)$. Now $r^{-1}(f)$ consists of three points $\tau_{1} \in I_{1}, \tau_{2} \in I_{2}$ and $\tau_{3} \in I_{3}$. Let $f_{1}=p\left(\tau_{1}\right), f_{2}=p\left(\tau_{2}\right)$ and $f_{3}=p\left(\tau_{3}\right)$. Join $f_{1}, f_{2}$ and $f_{3}$ pairwise by geodesics to bound a triangle, which we denote by $\alpha(\Delta)$, of perimeter no greater than $3 \delta+3$ as in Figure 2 . If $D$ is the Dehn function of $G$, we thus have $A(\alpha(\Delta)) \leq D(3 \delta+3)$. To finish the proof, therefore, it remains to show that the area of $\Delta-\alpha(\Delta)$ depends linearly on $\pi(\Delta)$.

Clearly it suffices to show that the area of each of these triangles depends linearly on $\pi(\Delta)$. So, consider $\lambda_{1}$, with sides $s_{1}=\left[g_{1}, f_{1}\right], s_{2}=\left[g_{1}, f_{3}\right]$ and $s_{3}=\left[f_{1}, f_{3}\right]$ (note that $\left|l\left(s_{1}\right)-l\left(s_{2}\right)\right| \leq 1$ and $\left.l\left(s_{3}\right) \leq \delta\right)$. We now subdivide $\lambda_{1}$ into further regions as follows. $I_{1}^{1}\left(l_{1}, l_{2}, l_{3}\right)$ and $I_{3}^{2}\left(l_{1}, l_{2}, l_{3}\right)$ are the segments of $\Delta_{A}\left(l_{1}, l_{2}, l_{3}\right)$ which map by $p$ onto $\left[g_{1}, f_{1}\right]$ and $\left[g_{1}, f_{3}\right]$. Suppose that the maximum of their lengths is $l_{4}$. Define $p_{i}^{1}=p(i)$ for $i \in I_{1}\left(l_{1}, l_{2}, l_{3}\right)$ if $i$ is an integer with $0 \leq i \leq l_{4}-1$ and $p_{i}^{2}=p\left(l_{3}-i\right)$ for $i \in I_{3}\left(l_{1}, l_{2}, l_{3}\right)$ if $i$ is an integer with $0 \leq i \leq l_{4}-1$. If $l\left(I_{1}\left(l_{1}, l_{2}, l_{3}\right)\right)=l(4)$ then define $p_{l_{4}}^{1}=p\left(l_{4}\right)$ and otherwise define $p_{l_{4}}^{1}=p\left(l_{4}-1\right)$, and make similar endpoint adjustments for the other segments. For each $i$ pick a geodesic $\eta_{i}$ between $p_{i}^{1}$ and $p_{i}^{2}$, and let $e_{j}^{i}$ be the edge from $p_{i}^{j}$ to $p_{i+1}^{j}$ for $j=1$ and 2 . Define $Q_{i}$ to be a least area quadrilateral bounded by $\eta_{i} * e_{i}^{2} * \eta_{i+1}^{-1} *\left(e_{i}^{1}\right)^{-1}$ for $0 \leq i \leq m-1$. Then
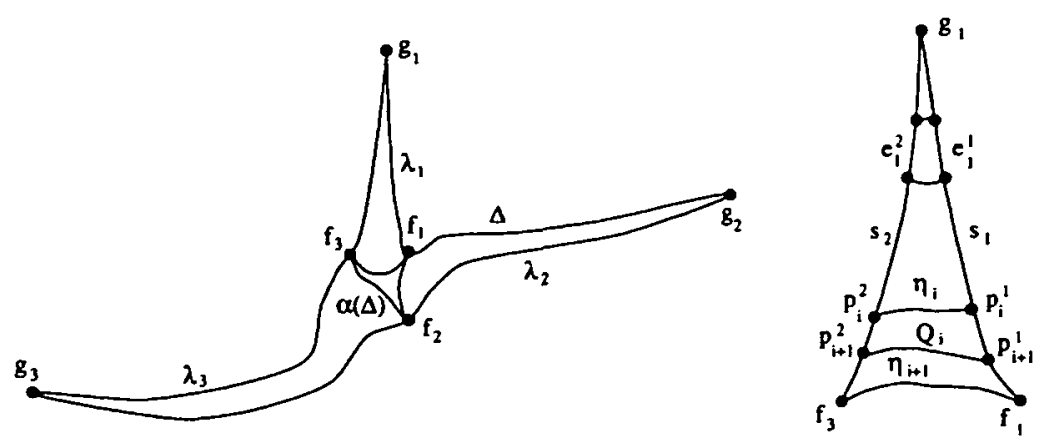

FIGURE 2: Dividing A Thin Triangle 


$$
\begin{aligned}
A\left(\lambda_{1}\right) & =\sum_{i=0}^{m-1} A\left(Q_{i}\right)+D(2 \delta+1) \\
& \leq m D(2 \delta+2)+D(2 \delta+1) \\
& \leq \pi(\Delta) D(2 \delta+2)+D(2 \delta+1),
\end{aligned}
$$

since $m \leq \pi(\Delta)$. So we can take $y(n)=3 D(2 \delta+2) n+D(3 \delta+3)+D(2 \delta+1)$, which is linear in $n$ as required.

The following example shows that the same property does not hold for slim triangles.

Example 2.9. Let $G$ be the free abelian group of rank 2 with the presentation $\langle a, b \mid[a, b]\rangle$. Define the triangle $\Delta_{n}$ to have vertices $e, a^{n}$ and $b^{n}$, and sides described by the words $a^{n}\left(a^{-1} b\right)^{n}$ from $e$ to $b^{n},\left(a^{-1} b\right)^{n} b^{-n}$ from $a^{n}$ to $e$ and $b^{-n} a^{n}$ from $b^{n}$ to $a^{n}$ (see Figure 3). Then $\Delta_{n}$ is a $(3,0)$-quasigeodesic 0 -slim triangle for all $n$. However, the perimeter of $\Delta_{n}$ is a linear function of $n$ but the area of $\Delta_{n}$ depends quadratically on $n$.

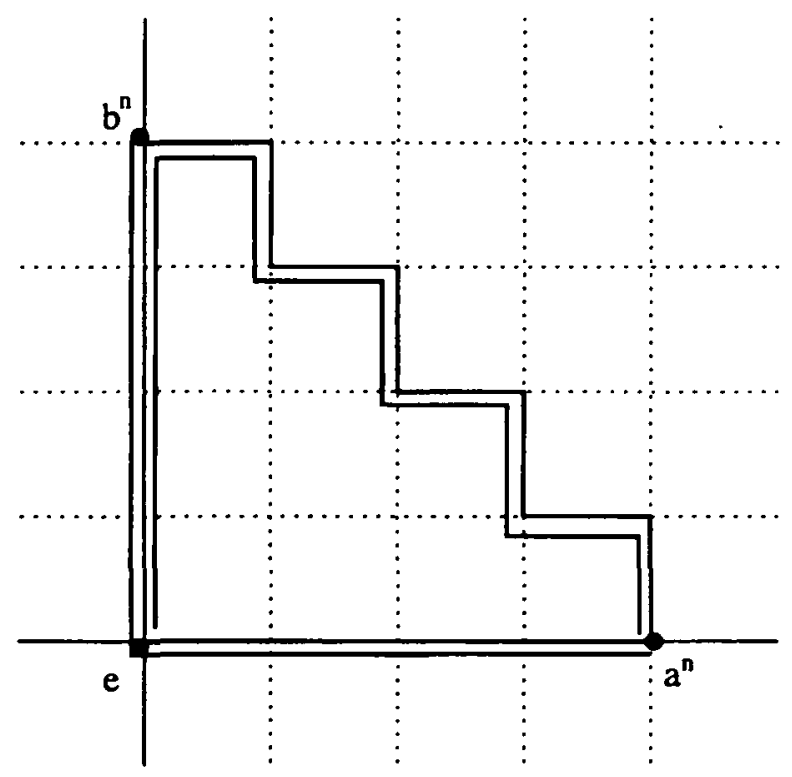

FIGURE 3: Slim Triangle with Quadratic Area 


\section{Thin and slim combings}

If $X$ is a graph then let $P(X)$ denote the set of finite paths in $X$. Whenever $p \in P(X)$ we shall write $i(p)$ for the initial vertex of $p$ and $t(p)$ for the terminal vertex of $p$.

Now let $G$ be a finitely generated group and let $S$ be a finite generating set for $G$.

Definition 3.1. A combing of a group $G$ with respect to a generating set $S$ is a map $\theta: G \rightarrow P\left(\Gamma_{s}(G)\right)$ such that for all $g \in G, i(\theta(g))=1_{G}$ and $t(\theta(g))=g$.

Note that we do not assume that the "fellow traveller property" of [3] holds for a combing.

We can equivariantly extend a combing $\theta$ of $G$ to a map $\hat{\theta}: G \times G \rightarrow P\left(\Gamma_{s}(G)\right.$ ) via the rule $\hat{\theta}\left(g_{1}, g_{2}\right)=g_{1} \theta\left(g_{1}^{-1} g_{2}\right)$. From now on, we shall also write $\theta$ for $\hat{\theta}$. Now if $\theta$ is a combing of $G$ and $g_{1}, g_{2}$ and $g_{3}$ are three elements of $G$, then we use the notation $\Delta_{0}\left(g_{1}, g_{2}, g_{3}\right)$ for the triangle

$$
\left(g_{1}, g_{2}, g_{3}, \theta\left(g_{1}, g_{2}\right), \theta\left(g_{2}, g_{3}\right), \theta\left(g_{3}, g_{1}\right)\right)
$$

We now introduce two types of constraint on the triangles of a combing. Since the definition of a thin triangle only applies to proper triangles, we define a combing $\theta$ of a group $G$ to be triangular if for all $g_{1}, g_{2}$ and $g_{3}$ in $G, \Delta_{0}\left(g_{1}, g_{2}, g_{3}\right)$ is a proper triangle.

Definition 3.2. Let $\delta \geq 0$. A combing (respectively triangular combing) $\theta$ of a group $G$ is $\delta$-slim (respectively -thin) if for all $g_{1}, g_{2}$ and $g_{3}$ in $G, \Delta_{0}\left(g_{1}, g_{2}, g_{3}\right)$ is a $\delta$-slim (respectively -thin) triangle. We say that $\theta$ is $\operatorname{sim}$ (respectively thin) if there exists $\delta \geq 0$ such that $\theta$ is $\delta$-slim (resp. -thin).

Proposition 3.3. If a combing $\theta$ of a group $G$ is triangular, then there exists a constant $K \geq 1$ such that for all $g_{1}$ and $g_{2}, l\left(\theta\left(g_{1}, g_{2}\right)\right) \leq K d\left(g_{1}, g_{2}\right)$.

Proof. Let $G$ be a finitely generated group with a finite generating set $S=\left\{s_{1}, \ldots, s_{p}\right\}$ and let $\theta$ be a triangular combing of $G$ with respect to $S$. Take $K$ to be $\max _{s \in S}\left\{l\left(\theta\left(1_{G}, s\right)\right)\right\}$. For $g_{1}$ and $g_{2}$ in $G$, let $w$ be a geodesic word in $S$ with $\bar{w}=g_{1}^{-1} g_{2}$. Suppose that $w=\prod_{j=1}^{m} s_{i j}^{n_{j}}$ where $m=d\left(g_{1}, g_{2}\right)$ and for each $j$ with $1 \leq j \leq m$, $n_{j} \in \mathbb{Z}-\{0\}$ and $1 \leq i_{j} \leq p$. Let $w_{k}=\prod_{j=1}^{k} s_{i_{j}}^{n_{j}}$ for $1 \leq k \leq m$ and let $w_{0}$ be the empty word. Then by repeated application of the triangle inequality we have

$$
\begin{aligned}
l\left(\theta\left(g_{1}, g_{2}\right)\right) & \leq \sum_{k=0}^{m-1} l\left(\theta\left(g_{1} \overline{w_{j}}, g_{1} \overline{w_{j+1}}\right)\right) \\
& \leq K m \\
& =K d\left(g_{1}, g_{2}\right) .
\end{aligned}
$$


Let $\lambda$ and $\mu$ be real numbers with $\lambda \geq 1$ and $\mu \geq 0$ and suppose that $p$ is a path in a graph. If for all subpaths $q$ of $p$ we have

$$
\frac{1}{\lambda} d(i(q), t(q))-\mu \leq l(q) \leq \lambda d(i(q), t(q))+\mu
$$

then we call $p$ a $(\lambda, \mu)$-quasigeodesic. If for a path $p$ there exist $\lambda \geq 1$ and $\mu \geq 0$ such that $p$ is a $(\lambda, \mu)$-quasigeodesic then we simply say that $p$ is a quasigeodesic.

Definition 3.4. A combing $\theta$ of a group $G$ is quasigeodesic if there exist $K \geq 1$ and $L \geq 0$ such that every combing line of $\theta$ is a $(K, L)$-quasigeodesic.

Note that the conclusion of Proposition 3.3 does not imply that $\theta$ is a quasigeodesic combing. Jean-Philippe Preaux has pointed out the following example due to Hamish Short.

Example 3.5. Consider the combing of $\mathbb{Z}=\langle x\rangle$ given by

$$
\theta\left(x^{n}\right)=x^{n}\left(x x^{-1}\right)^{n}
$$

for all $n \in \mathbb{Z}$ and extending equivariantly to give combing lines between all pairs of integers. Then $l\left(\theta\left(x^{n}\right)\right)=3 n$. Let $m_{1}, m_{2}$ and $m_{3}$ be three integers. Now

$$
\begin{aligned}
l\left(\theta\left(m_{1}, m_{3}\right)\right) & =3\left|m_{1}-m_{3}\right| \\
& =3\left|m_{1}-m_{2}+m_{2}-m_{3}\right| \\
& \leq 3\left|m_{1}-m_{2}\right|+3\left|m_{2}-m_{3}\right| \\
& =l\left(\theta\left(m_{1}, m_{2}\right)\right)+l\left(\theta\left(m_{2}, m_{3}\right)\right) .
\end{aligned}
$$

So $\theta$ is a triangular combing. The conclusion of the previous proposition is clearly satisfied by $\theta$, with $K=3$. But consider the subword $\left(x x^{-1}\right)^{n}$ of $\theta\left(x^{n}\right)$. The distance between the origin and the terminus of this path is 0 , but $l\left(\left(x x^{-1}\right)^{n}\right)=2 n$. So for all $L \geq 0$ and for all $K \geq 1$ there exists an element of $G$, e.g. $x^{L}$, such that $\theta\left(x^{L}\right)$ is not a $(K, L)$-quasigeodesic. Hence $\theta$ is not a quasigeodesic combing.

It is known that if there exists $\delta_{1}>0$ such that every geodesic triangle in a group $G$ is $\delta$-slim then there exists $\delta_{2}>0$, depending on $\delta_{1}$, such that every geodesic triangle in $G$ is $\delta_{2}$-thin (see e.g. [10]). If we no longer restrict to geodesic triangles, thin triangles are still slim, so if a group admits a thin combing then it admits a slim one.

Question. If a group admits a slim quasigeodesic combing then does it admit a thin combing? 


\section{Hyperbolicity and thin combings}

Reeves [9] has verified that $\mathbb{Z} \oplus \mathbb{Z}$ admits no thin combing, using work of Neumann and Shapiro which classifies biautomatic structures on free abelian groups [5]. Reeves has also asked the following question [8].

Question. Does admission of a thin combing characterise hyperbolicity of biautomatic groups?

We answer this question in the affirmative, and show that the hypothesis of biautomaticity is unnecessary. The usual concept of hyperbolicity requires all geodesic triangles to be $\delta$-thin, but here we show that for hyperbolicity, it is only necessary for one triangle per triple of points to be thin. Also the sides of the triangle no longer need to be geodesics. In this sense our main theorem is an "efficient" criterion for hyperbolicity.

First, recall that a group is said to satisfy a linear isoperimetric inequality if its Dehn function $D$ is bounded above by a linear function and that a finitely presented group satisfies a linear isoperimetric inequality if and only if it is hyperbolic (see [4] or [10]). We restrict to finitely presented groups because every finitely generated group $G$ satisfies a linear isoperimetric inequality if we include all words equal to the identity in $G$ as relators. A group is said to satisfy a subquadratic isoperimetric inequality if $\lim _{n \rightarrow \infty}\left(\frac{D(n)}{n^{2}}\right)=0$. The following result, originally due to Gromov [4], will play an important part in our analysis. Proofs have been given by Ol'Shanskii [6], Papasoglu [7] and Bowditch [2].

Theorem 4.1. If a group satisfies a subquadratic isoperimetric inequality then it satisfies a linear one (and so is hyperbolic).

We can now state our main theorem.

Theorem 4.2. A finitely presented group admits a thin combing if and only if it is hyperbolic.

Proof. Clearly every hyperbolic group $G$ admits a thin combing (in every Cayley graph with respect to a finite generating set, every geodesic combing is thin).

Conversely, let $G$ be a finitely presented group admitting a $\delta$-thin combing $\theta$. We are going to show that $G$ verifies a subquadratic isoperimetric inequality. Let $\phi: F(S) \rightarrow G$ be a choice of generators for $G$, where $S$ is finite, and let $w$ be a word of length $n_{w}$ in $S$ whose image is equal to the identity in $G$. In $\Gamma_{s}(G), \bar{w}$ represents a loop of length $n_{w}$, originating and terminating at $1_{G}$. We may assume that $\bar{w}$ is a simple loop since, if not, $\bar{w}$ may be divided into several loops $w_{1}, \ldots, w_{n}$ with $A(\bar{w}) \leq \sum_{i=1}^{n} A\left(w_{i}\right)$.

We now define a finite sequence of elements of $G$. Let $n$ be the smallest power of 2 such that $n \geq n_{w}$. Then $n \leq 2 n_{w}$. For $0 \leq i \leq n_{w}$, let $h_{i}=\bar{w}(i)$ and for $n_{w} \leq i \leq n$, define $h_{i}=1_{G}$. We now use the combing lines of $\theta$ to subdivide the loop $\bar{w}$. Let $g=h_{\frac{n}{2}}, g_{1}=h_{h}$ 


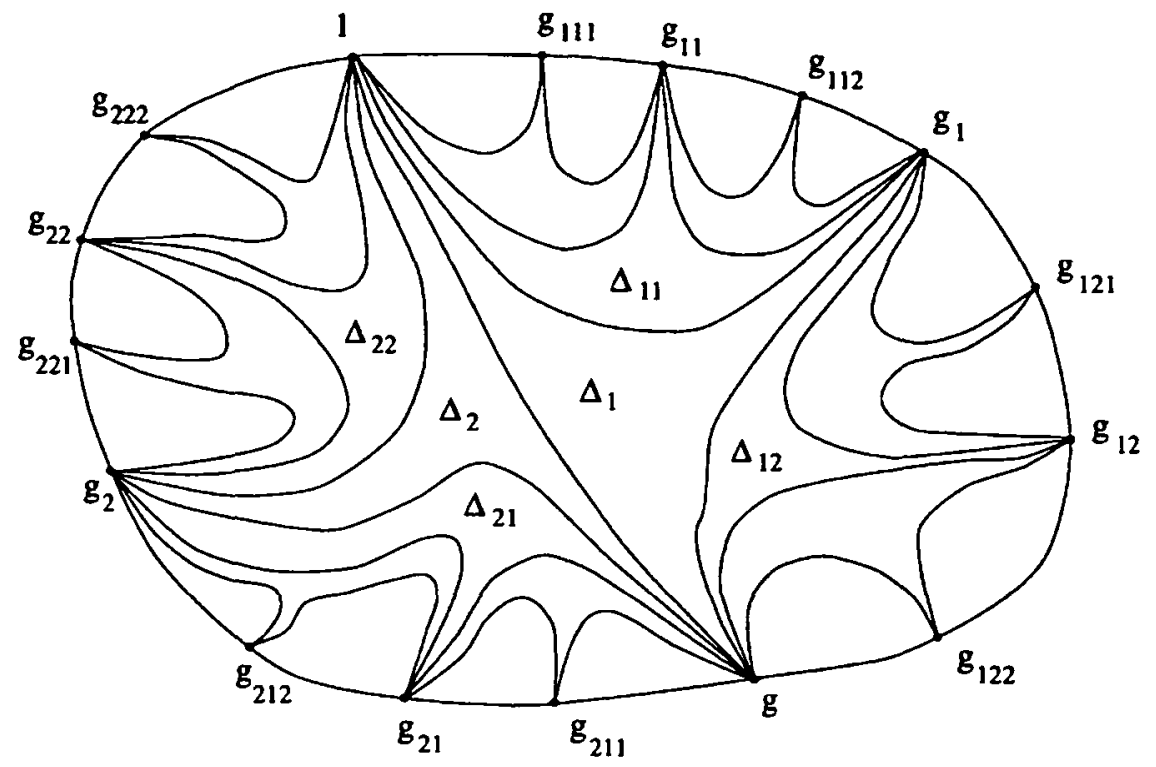

FIGURE 4: A Thin Combing Implies Hyperbolicity

and $g_{2}=h_{\frac{3 n}{4}}$ and define the triangles $\Delta_{1}=\Delta_{0}\left(1_{G}, g_{1}, g_{2}\right)$ and $\Delta_{2}=\Delta_{0}\left(g, g_{2}, 1_{G}\right)$. We next construct four more triangles on the sides of $\Delta_{1}$ and $\Delta_{2}$. Let $g_{11}=h_{\frac{n}{8}}, g_{12}=h_{\frac{3 n}{8}}, g_{21}=h_{\frac{5 n}{8}}$ and $g_{22}=h_{\frac{7 n}{8}}$, and define $\Delta_{11}=\Delta_{0}\left(1_{G}, g_{11}, g_{1}\right), \Delta_{12}=\Delta_{0}\left(g_{1}, g_{12}, g\right), \Delta_{21}=\Delta_{0}\left(g, g_{21}, g_{2}\right)$ and $\Delta_{22}=\Delta_{0}\left(g_{2}, g_{22}, 1_{G}\right)$. The process of subdividing is continued for each $1 \leq p \leq \log _{2}(n)-1$ to obtain group elements $g_{i_{1} \ldots i_{p}}$ and triangles $\Delta_{i_{1} \ldots i_{p}}$ for $i_{j}=1$ and 2 , as in Figure 4.

Let $Q_{p}$ be the set of triangles introduced at the $p^{\text {th }}$ stage of the subdivision and let $Q$ be the union of all the triangles in the subdivision. We then have

$$
A(Q) \leq \sum_{p=1}^{\log _{2}(n)-1} \sum_{T \in Q_{p}} A(T) .
$$

Suppose that $K$ is as in Proposition 3.3 and that $y$ is a linear function as in Proposition 2.8. If $T \in Q_{p}$ then we have $\pi(T) \leq \frac{K n}{2^{p-1}}$ and

$$
A(T) \leq \frac{K y(n)}{2^{p-1}}
$$

Now $Q_{p}$ contains $2^{p}$ triangles and if $D$ is the Dehn function of $\Gamma_{s}(G)$ then we have 


$$
\begin{aligned}
A(w) & \leq A(Q)+n D(K+1) \\
& \leq \sum_{p=1}^{\log _{2}(n)-1} \frac{2^{p} \cdot K y(n)}{2^{p-1}}+n D(K+1) \\
& \leq 2 K y(n) \log _{2}(n)+n D(K+1) \\
& \leq 4 K y\left(n_{w}\right) \log _{2}\left(2 n_{w}\right)+2 n_{w} D(K+1) .
\end{aligned}
$$

Thus $G$ satisfies an isoperimetric inequality which is $O\left(n \log _{2} n\right)$. Since this is subquadratic, $G$ is hyperbolic by Theorem 4.1 .

Note that the homogeneity (as metric spaces) of Cayley graphs, along with the equivariance of combings, does not play an important role in the arguments. In fact, it is possible to generalise the above theorem to path-metric spaces, using a formulation of area due to Bowditch [2]. This is described in the author's Ph.D. thesis [1].

Acknowledgement. I would like to thank my Ph.D. supervisor David Epstein for useful conversations during the development of this work. I am also grateful to Lawrence Reeves, whose question inspired the main result, to Jean-Philippe Preux, who noticed several minor errors in a preprint version and to Martin Bridson and Derek Holt for carefully reading my thesis and suggesting that some quite subtle points needed correcting.

\section{REFERENCES}

1. M. BAtTY, Geometric Characterisations of Groups (Ph.D. Thesis, 1997, University of Warwick).

2. B. H. BowDitch, A short proof that a subquadratic isoperimetric inequality implies a linear one, Michigan Math. J. 42 (1995), 103-107.

3. D. B. A. Epstein, J. W. Cannon, D. F. Holt, S. V. F. Levy, M. S. Paterson and W. P. Thurston, Word Processing in Groups (Jones and Bartlett Publishers, 1992).

4. M. Gromov, Hyperbolic Groups, in Essays in Group Theory (ed. S. Gersten, MSRI Publications No. 8, Springer-Verlag, 1988), 75-263.

5. W. D. Neumann and M. Shapiro, Equivalent automatic structures and their boundaries, Internat. J. Algebra Comput. 2 (1992), 443-469.

6. A. Yu OlshansKII, Hyperbolicity of Groups with a Subquadratic isoperimetric Inequality, Internat. J. Algebra Comput. 1 (1991), 281-289.

7. P. Papasoglu, On the sub-quadratic isoperimetric inequality, in Geometric Group Theory (Columbus, OH, 1992) (Ohio State Univ. Math. Res. Inst. Publ., 3, de Gruyter, Berlin, 1995), 149-157.

8. L. REEVES, private communication.

9. L. ReEves, unpublished work.

10. H. Short (ed.), Notes on Word Hyperbolic Groups, in Group Theory from the Geometrical Viewpoint (eds. E. Ghys, A. Haefliger and A. Verjovsky, World Scientific, Singapore, 1991), 3-63.

Mathematics InSTITUTE

UNIVERSITY OF WARWICK

COVENTRY

ENGLAND 